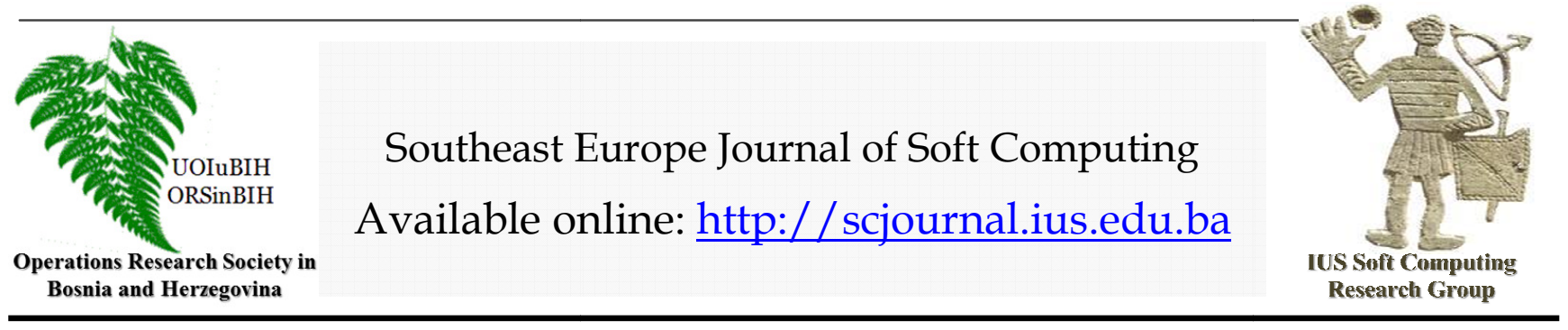

\title{
A Review on Image Enhancement Techniques
}

\author{
Haris Ačkar, Ali Abd Almisreb \\ Faculty of Engineering and Natural Sciences, \\ International University of Sarajevo, \\ Hrasnicka cesta 15, Ilidža 71210 Sarajevo, \\ Bosnia and Herzegovina \\ haris_ackar@hotmail.com
}

\author{
Mohamed A. Saleh \\ Faculty of Electrical Engineering, \\ Universiti Teknologi MARA (UiTM), \\ Malaysia
}

\section{Article Info}

\section{Article history:}

Article received on 13 January 2019 Received in revised form 25 February 2019

\section{Keywords:}

Enhancement;underwater images, medical images, deffoging algorithms, infrared images, heuristic methods.

\begin{abstract}
Image Enhancement is one of the most important and complex techniques in image processing technology. The main aim of image enhancement is to improve the visual appearance on an image and to offer a better representation of the image for Computer Vision Algorithms. In this paper,we is covered a few application fields of image enhancement with various images like grayscale, color, infrared and even with videos. The main objective of this paper is to highlight the drawbacks of the state of the art image enhancement techniques.
\end{abstract}

\section{INTRODUCTION}

Image enhancement is one of the preprocessing steps in various computer vision applications. It is the process of improving the quality of the image without any information loss. For example in medical images [1], contrast enhancement is mostly used for increasing the quality of any image. The basic importance of the contrast enhancement is to improve the pixel brightness in medical application. A lot of methods are introduced in research papers, and every method has one or few application fields. Depend on the field application, types of images, grayscale or color images, we need to choose the proper method for image enhancement. In this paper will be presented state of the art techniques for image enhancement of grayscale and color images. A short description of those methods will be provided and their field of application. Every Computer Vision algorithm requires high-visibility input images in order to produce some output. However, in most situations, images are taken in some specific conditions like low-light. Therefore, we need to enhance those images before further processing in order to have correct results from Computer Vision algorithms. In general, image enhancement methods need to ,correct" input images for specific purpose so that those images can be more suitable for specific Computer Vision Algorithms. 
This paper is organized into 8 sections, Section 1 gives an overview of the paper. It describes the importance of image enhancement in image processing. Section 2 gives a literature review for image enhancement techniques for underwater images. Section 3 gives a literature review for image enhancement techniques for medical images. Section 4 gives a short review of video and image defogging algorithms. Section 5 gives an overview of techniques for infrared image enhancement and sections 6 gives a literature review for contrast enhancement. Section 7 gives a short review of Haze visibility enhancement of images. Section 8 gives a short review of latest image enhancement techniques from 2018 and 2019.

\section{IMAGE ENHANCEMENT TECHNIQUES FOR UNDERWATER IMAGES}

Underwater images are corrupted due to scatters and amalgamation, resulting in low contrast and color distortion [2]. There are many image enhancement techniques such as white balance, color correction, histogram equalization, and fusion-based methods [3]. Various image enhancement techniques can be found in the literature. Hue variations can be minimized by wavelength compensation [4]. Also, underwater images have low perceivability, small divergence and lessening hues [5]. To enhance underwater images, some authors propose unsupervised digital image color equalization [6]. Other authors use histogram modifications techniques to increase the quality of underwater image $[7,8]$, and there are also researchers who purposes algorithms to reduce underwater perturbations and to improves image quality $[9,10]$.

\section{IMAGE ENHANCEMENT TECHNIQUES FOR MEDICAL IMAGES}

In the medical images, the main role plays contrast enhancement to increase the quality of an image [11]. Edge detection is also playing an important role in medical imaging because all information has preserved in edges. In literature can be found a lot of interesting image enhancement techniques for medical devices, classic but also and fuzzy approaches. Some researchers propose fuzzy hyperbolization to increase the clarity of the image [12]. There are also techniques for medical images using type-II fuzzy set [13]. The detailed description of type-II fuzzy set can be found in [14]. Survey paper like [15] presenting a detailed overview of image enhancement using fuzzy techniques. The main advantage of those techniques is to enhance the contrast and improve the quality of the image. A lot of authors are proposing various medical image enhancement techniques using type-II fuzzy approach. The [15] proposes type-II fuzzy computing techniques for image enhancement. The [16] proposes Edge detection method based on type-II fuzzy system for color images. At the end, papers like [17] can be found in literature, those papers describe the development of improved fuzzy rulebased edge detection technique.

\section{VIDEO AND IMAGE DEFOGGING ALGORITHMS}

Videos and images acquired by a visual system are seriously degraded under hazy and foggy weather, which will affect the detection, tracking, and recognition of targets. In that purpose a lot of video and image defogging algorithms is created. The images acquired by the camera in foggy and hazy weather is degraded and usually has low contrast and poor visibility [18]. Some of the interesting fields of application for image defogging algorithms are to enhance the visibility of the vehicle visual system, which can effectively prevent car accidents [19]. Some papers use a physical imaging model based on the atmospheric scattering phenomenon for image defogging [20, 21]. Many improved defogging algorithms for unmanned surface vehicle visual system are based on the physical model for outdoor scenes [22-26]. In past years, some institutions have done research on image defogging and obtained good results. NASA has studied the image enhancement and defogging algorithm since 1995, and their research has made a great contribution to the field of image enhancement based on the Retinex theory. Their algorithms can greatly enhance the visibility of an image acquired under bad weather conditions, such as smoke, haze, underwater, night or low illumination conditions [26-30].

\section{IMAGE ENHANCEMENT OF INFRARED IMAGES}

In this section, it will be provided a survey of enhancement of infrared images with two spatial and spatiotemporal homomorphic filtering algorithms based on infrared imaging model. The Wavelength of infrared light is longer than of visible light, the visible red light has wavelength of 0.74 micrometers at the end of visible spectrum, infrared light has 300 micrometers wide wavelength band startng from 0.74 micrometers. These wavelengths correspond to a frequency range of approximately 1 to $400 \mathrm{THz}$ [31]. Spatial and spatiotemporal homomorphic filtering algorithms are designed. The spatiotemporal homomorphic filter uses the temporal information provided by the image sequence so that an enhancement is faster than that obtained by utilizing the spatial homomorphic filtering. STHF will spend less time and iterations to compute a resulting image from a similar degree of convergences the enhance images are in general not good as those from SHF. Some authors suggest using Wavelets for edge detection and as approximate matched filters. Wavelets as edge detectors assume that target edges on image differ in some way from clutter edges $[32,33]$. In some cases, a priori knowledge of target is important in order to do scale separation [34]. Also, 
44 H. Ackar et al./ Southeast Europe Journal of Soft Computing Vol.8 No.1 March 2019 (42-48)

wavelets can be designed to function as approximate matched filters [35].

\section{CONTRASTENHANCEMENT TECHNIQUES}

Contrast is the visual difference that makes an object distinguishable from the background and other objects. Contranst enhancement techniques are changing pixel intensitiy of the input imate in order to utilize maximum possible bins. It has been an active research topic since the early days of computer vision and digital image processing. In this section, the focus will be on contrast enhancement techniques for effective contrast enhancement. Most of the researchers are focused on histogram-based contrast enhancement techniques. Histogram based techniques, like Homomorphic Filtering, are used to enhance the low contrast for medical images [36]. In the literature can be found modified versions of the hyperbolic algorithm for contrast enhancement. These modified algorithm are suitable for enhancement of magnetic resonance images. This technique uses controlled fashion of the gray level which is streching on whole image [37]. There are also contrast enhancement techniques based on Fuzzy techniques. Fuzzy techniques can manage imperfectness of an image modeled as the uncertainty in the image. Fuzzy method for contrast enhancement can be divided into three stages, like classic fuzzy system: fuzzification, modification of membership functions and defuzzification of the image [38]. In the literature can be found an automatic methods for contrast enhancement. For example, by grouping the histogram components of a low-contrast image into a proper number of bins according to a selected criterion, then redistribute these bins uniformly over the grayscale. The last step is to ungroup the previously grouped graylevels. This technique is known as gray-level grouping (GLG) [39, 40]. Some authors propose an extension of histogram equalization. With this approach, it is possible to have independent histogram equalizations for two subimages found by decomposing the input image based on its mean value. Resulting equalized sub-images are bounded by each other around the input mean. This approach has a great geature, it maintains the mean brightness of given input images significantly [41].

\section{VII. HAZE VISIBILITY ENHANCEMENT OF IMAGES}

The haze is the most common real-world phenomena caused by atmospheric particles. Images captured in hazy scenes suffers from visibility degradation and significant reduction of contrast. Recovering scenes from hazy images can be critical for image processing and computer vision algorithms. Algorithms for haze-free photographs are developed because consumers want a clear visual content when they are shooting target objects or landscapes. The floating particles in the atmosphere are absorbing and scattering light in the enviroment, because of this scattering and absorption we have degradation in hazy images. This scattering and absorption reduce the direct transmission of the light from the scene to the camera. The attenuated direct transmission causes the intensity from the scene to be weaker, while the airlight causes the appearance of the scene to be washed out [42]. The one of the earliest methods to analyze images of scenes captured in scattering media is to extract scene depth by exploiting the presence of the atmospheric scattering effects [43]. The methods proposed in [44] does not assume that the haze-free image is provided. These two methods are pioneers in dealing with atmospheric particles. Some methods for haze enhancement uses different base for enhancement like multiple images, polarizing filters, known depth and singleimage. The multiple images methods uses multiple images of same scene taken in different hazy conditions. With the assumption that images are taken from the same scene, they are sharing same color of atmospheric light but they have a different direct transmission of colors. From this two planes can be formed in the RGB space that intersect each other. In [45] is presented how to estimate the atmospheric light sith this intersect, which is similar to [46] for estimating a light color from specular reflection. For methods with known depth is proposed several algorithms based on a single input image and they requires some user interaction [47]. The first method from this paper requires from user to select a region with less haze and region with more haze of the same reflection as the first one's. From these inputs, proposed algorithm can calculate resulting image and dehaze hazy pixels. The second method asks user to indicate vanishing point and to input the maximum and minimum distance from the camera. This information is used to interpolate the distance to estiamte the clear secene between. In the paper [48] is proposed a framework for contrast enhnacement of images taken in a vehicle.

\section{LATEST IMAGE ENHANCEMENT TECHNIQUES}

The latest image enhnacement technques can be devided into two groups, heuristic and classic image enhancement techniques. In first part of this chapter, it will be given review about novel classic image enhancement techniques and after that it will be given review about heuristic image enhancement techniques. One of the latest classic algorithms for contours detectin in thermal images is based on theory of sampling Kantorovich operators and on analysis of the histogram of the enhanced thermographic image [49]. Contrast Enhancement is an important step for the analysis of microscopy images. In paper [50] is proposed groundbrakeing design of the Phase Contrast Microscopy Framework. The proposed image enhnacement framework transforms the changes in image phase into the variations of magnitude to enhance the structural details of the image and to improve visibility. In paper [51] is proposed algorithm for image enhancing approach for transforming dark images into lightened scenes. This 
approach uses classical color transfer method to obtain first order statistics from a target image and transfer them to a dark input by modifying its hue and brightness. This method can be used as a preprocessing step in order to improve the recognition and interpretation of dark imagery in a wide range of applications. Remote sensing images often suffer low contrast, and the efficiency and robustness of contrast enhancement. In the paper [52] is proposed improved adaptive contrast enhancement method based on histogram compacting transform. In the papers $[53,54]$ are presented low-lightning image enhancement based on Retinex model. The different Retinex models are proposed in order to enhance illumination and reflectance. New image enhnacement method based on Nonsubsampled Contourlet Transform is proposed in paper [55]. In this paper is presented contourlet transform as an extension of the wavelet transform that provides a multi-resolution and multi-direction analysis for two dimensional images. In the paper [56] is presented a high-resolution image enhancement wavelet-based algorithm for edge smoothness of an Satelite Image. The proposed algorithm apply the three-level discrete wavelet transform and compute the output of the algorithm. In order to improve contrast and restore color for underwater images without suffering from insufficient details and color cast, in the paper [57] is proposed a fusion algorithm for different color spaces based on contrast limited adaptive histogram equalization.

In order to have an effective colour enhancement framework for statistical and logarithmic image processing enhancement algorithms, the paper [58] proposes approach of utilizing the fusion of partial, multiple computed luminance channels with colour image channel statistics obtained from the input colour image for adaptive color enhancement. The Unmanned Aerial Vehicles are widely used for capturing images in border area surveillance, disaster intensity monitoring, etc. In the paper [59] is presented usage of Firefly Algorithm in order to enhance aerial images taken by a Mini Unmanned Aerial Vehicle via optimizing the value of certain parameters. For Smart City applications it is crucial to have a good pre-processing technique for infrared image enhancement. Existing grayscale mapping-based algorithms always suffer from over-enhancement of the background, noise amplicifation and brightness distortion. In the paper [60] is proposed an adaptive histogram partition and brightness correction in order to correct above mentioned problems.

In the paper [61] are proposed two novel histogram based image enhancement algorithms. The proposed algorithms provide the way to control the brightness and contrast of enhanced image by adjusting the parameters. After classic image enhancement techniques, the novel huristic methods will be described. Low-light is a challenging enviroment for image processing. In the paper [62] is proposed method for low-light image enhancement using Gaussian Process and Convolutional Neural Network. In the papers $[63,64]$ are proposed image enhancement techniques using biologically inspired artificial Bee Colony Algorithms. In this papers image constrast enhnacement is considered as an optimization problem and the artificial bee colony algorithm is utilized to find the optimal solution for this optimization problem. In the paper [65] can be found a survay on Nature-Inspired optimization algorithms and their application in image enhancement domain. Single image contrast enhancement methods are used to adjust the tone curve to correct the contrast of an input image. In the paper [66] is proposed learning algorithm of convolutional neural network to train a single image contrast enhancer. In the paper [67] is proposed a deep learning neural network with purpose of obtaining enhanced representations of the sequences for visual odometry. After initial results, it is proposed a reduced size of convolutional neural network for faster computation. In the paper [68] is propsoed Learned Perceptual Image Enhancement technique. The two main contributions of this paper are presenting state of the art predictor that encompasses several aspects of human perceptual preferences and the researchers uses neural image asessment as a perceptual loss for image enhancement tasks.

\section{CONCLUSION}

Image enhancement techniques change images to provide a better representation of the information encapsulated in the image. In this paper is presented a review for various fields of image enhancement. For every purpose like underwater imaging or medical images, there are different algorithms and techniques suitable for image enhancement. As we can see from this review paper, and also from other review papers, there is no universal image enhancement technique, the most important reason for that is the fact that there are a lot of different factors, for example, fogg in images and videos, or the fact that medical images are mostly grayscale images.

\section{REFERENCES}

[1] Rana, S. B., \& Rana, S. B. (2015). A Review of Medical Image Enhancement Techniques for Image Processing. International Journal of Current Engineering and Technology, 5(2), 1282-1286.

[2] Sandbhor, B., \& Kharat, G. U. (2015). A review on Underwater Image Enhancement Techniques. Int. J. Advanced Research in Computer Science and Software Engineering, 5(5), 676-680.

[3] Ancuti, C., Ancuti, C. O., Haber, T., \& Bekaert, P. (2012, June). Enhancing underwater images and videos by fusion. In 2012 IEEE Conference on Computer Vision and Pattern Recognition (pp. 8188). IEEE.

[4] Sathya, R., \& Bharathi, M. (2015). Enhancement of Underwater Images Using Wavelength Compensation Method. International Journal of Innovative Research in Computer and Communication Engineering, 3(3), 1829-1835.

[5] Talwar, S., \& Kochher, R. (2015). Evaluation of Underwater Image Dehazing Techniques. 
46 H. Ackar et al./ Southeast Europe Journal of Soft Computing Vol.8 No.1 March 2019 (42-48)

International Journal for Science and Emerging Technologies with Latest Trends, 21(1), 11-13.

[6] Rizzi, A., Gatta, C., \& Marini, D. (2003). A new algorithm for unsupervised global and local color correction. Pattern Recognition Letters, 24(11), 1663-1677.

[7] Iqbal, K., Salam, R. A., Osman, A., \& Talib, A. Z. (2007). Underwater Image Enhancement Using an Integrated Colour Model. IAENG International Journal of Computer Science, 34(2).

[8] Iqbal, K., Odetayo, M., James, A., Salam, R. A., \& Talib, A. Z. H. (2010, October). Enhancing the low quality images using unsupervised colour correction method. In 2010 IEEE International Conference on Systems, Man and Cybernetics (pp. 1703-1709). IEEE.

[9] Bazeille, S., Quidu, I., Jaulin, L., \& Malkasse, J. P. (2006, October). Automatic underwater image preprocessing. In CMM'06 (p. Xx).

[10] Bazeille, S. (2008). Vision sous-marine monoculaire pour la reconnaissance d'objets (Doctoral dissertation, Brest).

[11] Kaur, R., Chawla, M., Khiva, N. K., \& Ansari, M. D. (2017). On Contrast Enhancement Techniques for Medical Images with Edge Detection: A Comparative Analysis. Journal of Telecommunication, Electronic and Computer Engineering (JTEC), 9(3-6), 35-40.

[12] Hartati, S., Harjoko, A., \& Nickerson, B. G. (2009). Fuzzy hyperbolization image enhancement and artificial neural network for anomaly detection.

[13] Chaira, T. (2012). A rank ordered filter for medical image edge enhancement and detection using intuitionistic fuzzy set. Applied soft computing, 12(4), 1259-1266.

[14] Karnik, N. N., Mendel, J. M., \& Liang, Q. (1999). Type-2 fuzzy logic systems. IEEE transactions on Fuzzy Systems, 7(6), 643-658.

[15] Sesadri, U., \& Nagaraju, C. (2015). TYPE2 FUZZY SOFT COMPUTING TECHNIQUE FOR IMAGE ENHANCEMENT. International Journal of Computer Science and Information Security, 13(11), 94.

[16] Ansari, M. D., Ghrera, S. P., \& Tyagi, V. (2014). Pixel-based image forgery detection: A review. IETE journal of education, 55(1), 40-46.

[17] Ansari, M. D., \& Ghrera, S. P. (2018). Intuitionistic fuzzy local binary pattern for features extraction. International Journal of Information and Communication Technology, 13(1), 83-98.

[18] Sharma, R., \& Chopra, V. (2014). A review on different image dehazing methods. International Journal of Computer Engineering and Applications, 6(3).

[19] Hautière, N., Tarel, J. P., \& Aubert, D. (2007, June). Towards fog-free in-vehicle vision systems through contrast restoration. In 2007 IEEE
Conference on Computer Vision and Pattern Recognition (pp. 1-8). IEEE.

[20] Narasimhan, S. G., \& Nayar, S. K. (2002). Vision and the atmosphere. International journal of computer vision, 48(3), 233-254.

[21] Narasimhan, S. G., \& Nayar, S. K. (2001, December). Removing weather effects from monochrome images. In IEEE computer society conference on computer vision and pattern recognition (Vol. 2, pp. II-186). IEEE Computer Society; 1999.

[22] John, J., \& Wilscy, M. (2008, September). Enhancement of weather degraded video sequences using wavelet fusion. In 2008 7th IEEE International Conference on Cybernetic Intelligent Systems (pp. 1-6). IEEE.

[23] Ramya, C., \& Rani, D. S. S. (2012). Contrast enhancement for fog degraded video sequences using BPDFHE. Int. J. Comput. Sci. Inf. Technol, 3(2), 3463-3468.

[24] Xu, Z., Liu, X., \& Chen, X. (2009, December). Fog removal from video sequences using contrast limited adaptive histogram equalization. In 2009 International Conference on Computational Intelligence and Software Engineering (pp. 1-4). IEEE.

[25] Lin, Z., \& Wang, X. (2012). Dehazing for image and video using guided filter. Appl. Sci, 2, 123127.

[26] SINGH RAJPUT, G., \& RAHMAN, Z. U. (2008). Hazard Detection on Runways using Image processing Techniques. In Proceedings of SPIE, the International Society for Optical Engineering (pp. 69570D-1). Society of Photo-Optical Instrumentation Engineers.

[27] Jobson, D. J., Rahman, Z. U., \& Woodell, G. A. (1996, January). Retinex image processing: Improved fidelity to direct visual observation. In Color and Imaging Conference (Vol. 1996, No. 1, pp. 124-125). Society for Imaging Science and Technology.

[28] Rahman, Z. U., Jobson, D. J., \& Woodell, G. A. (1996, November). Multiscale retinex for color rendition and dynamic range compression. In Applications of Digital Image Processing XIX (Vol. 2847, pp. 183-192). International Society for Optics and Photonics.

[29] Rahman, Z. U., Woodell, G. A., \& Jobson, D. J. (1997). A comparison of the multiscale retinex with other image enhancement techniques.

[30] Rahman, Z. U., Jobson, D. J., Woodell, G. A., \& Hines, G. D. (2005, September). Image enhancement, image quality, and noise. In Photonic Devices and Algorithms for Computing VII (Vol. 5907, p. 59070N). International Society for Optics and Photonics.

[31] Qi, H., \& Head, J. F. (2001). Asymmetry analysis using automatic segmentation and classification 
47 H. Ackar et al./ Southeast Europe Journal of Soft Computing Vol.8 No.1 March 2019 (42-48)

for breast cancer detection in thermograms. In 2001 Conference Proceedings of the 23rd Annual International Conference of the IEEE Engineering in Medicine and Biology Society (Vol. 3, pp. 2866-2869). IEEE.

[32] Kuruganti, P. T., \& Qi, H. (2002). Asymmetry analysis in breast cancer detection using thermal infrared images. In Proceedings of the Second Joint 24th Annual Conference and the Annual Fall Meeting of the Biomedical Engineering Society][Engineering in Medicine and Biology (Vol. 2, pp. 1155-1156). IEEE.

[33] Scales, N., Kerry, C., \& Prize, M. (2004, September). Automated image segmentation for breast analysis using infrared images. In The 26th Annual International Conference of the IEEE Engineering in Medicine and Biology Society (Vol. 1, pp. 1737-1740). IEEE.

[34] Zhang, C. J., Yang, F., Wang, X. D., \& Zhang, H. R. (2005, August). An efficient non-linear algorithm for contrast enhancement of infrared image. In 2005 International Conference on Machine Learning and Cybernetics (Vol. 8, pp. 4946-4951). IEEE.

[35] Andreone, L., Antonello, P. C., Bertozzi, M., Broggi, A., Fascioli, A., \& Ranzato, D. (2002, September). Vehicle detection and localization in infra-red images. In Proceedings. The IEEE 5th International Conference on Intelligent Transportation Systems (pp. 141-146). IEEE.

[36] Agarwal, T. K., Tiwari, M., \& Lamba, S. S. (2014, February). Modified histogram based contrast enhancement using homomorphic filtering for medical images. In 2014 IEEE International Advance Computing Conference (IACC) (pp. 964968). IEEE.

[37] Chong, S. S., Sim, K. S., \& Nia, M. E. (2013, October). Modified HL contrast enhancement technique for breast MR images. In 2013 IEEE International Conference on Signal and Image Processing Applications (pp. 360-364). IEEE.

[38] Mahashwari, T., \& Asthana, A. (2013). Image enhancement using fuzzy technique. International Journal of Research in Engineering Science and Technology, 2(2), 1-4.

[39] Chen, Z., Abidi, B. R., Page, D. L., \& Abidi, M. A. (2006). Gray-level grouping (GLG): an automatic method for optimized image contrast Enhancement-part I: the basic method. IEEE transactions on image processing, 15(8), 22902302.

[40] Chen, Z., Abidi, B. R., Page, D. L., \& Abidi, M. A. (2006). Gray-level grouping (GLG): an automatic method for optimized image contrast enhancement-part II: the variations. IEEE Transactions on Image Processing, 15(8), 23032314.

[41] Kim, Y. T. (1997). Contrast enhancement using brightness preserving bi-histogram equalization. IEEE transactions on Consumer Electronics, 43(1), $1-8$.

[42] Li, Y., You, S., Brown, M. S., \& Tan, R. T. (2017). Haze visibility enhancement: A survey and quantitative benchmarking. Computer Vision and Image Understanding, 165, 1-16.

[43] Cozman, F., \& Krotkov, E. (1997, June). Depth from scattering. In cvpr (Vol. 31, pp. 801-806).

[44] Nayar, S. K., \& Narasimhan, S. G. (1999). Vision in bad weather. In Proceedings of the Seventh IEEE International Conference on Computer Vision (Vol. 2, pp. 820-827). IEEE.

[45] Narasimhan, S. G., \& Nayar, S. K. (2000). Chromatic framework for vision in bad weather. In Proceedings IEEE Conference on Computer Vision and Pattern Recognition. CVPR 2000 (Cat. No. PR00662) (Vol. 1, pp. 598-605). IEEE.

[46] Tominaga, S., \& Wandell, B. A. (1989). Standard surface-reflectance model and illuminant estimation. JOSA A, 6(4), 576-584.

[47] Narasimhan, S. G., \& Nayar, S. K. (2003, October). Interactive (de) weathering of an image using physical models. In IEEE Workshop on color and photometric Methods in computer Vision (Vol. 6, No. 6.4, p. 1). France.

[48] Hautière, N., Tarel, J. P., \& Aubert, D. (2007, June). Towards fog-free in-vehicle vision systems through contrast restoration. In 2007 IEEE Conference on Computer Vision and Pattern Recognition (pp. 1-8). IEEE.

[49] Asdrubali, F., Baldinelli, G., Bianchi, F., Costarelli, D., Rotili, A., Seracini, M., \& Vinti, G. (2018). Detection of thermal bridges from thermographic images by means of image processing approximation algorithms. Applied Mathematics and Computation, 317, 160-171.

[50] Cakir, S., Kahraman, D. C., Cetin-Atalay, R., \& Cetin, A. E. (2018). Contrast enhancement of microscopy images using image phase information. IEEE Access, 6, 3839-3850.

[51] Cepeda-Negrete, J., Sanchez-Yanez, R. E., CorreaTome, F. E., \& Lizarraga-Morales, R. A. (2018). Dark image enhancement using perceptual color transfer. IEEE Access, 6, 14935-14945.

[52] Chen, J., Liu, J., Zhou, C., Zhu, F., Chen, T., \& Zhang, H. (2018, August). An automatic image enhancement method based on the improved HCTLS. In 2018 10th IAPR Workshop on Pattern Recognition in Remote Sensing (PRRS) (pp. 1-6). IEEE.

[53] Gao, Y., Hu, H. M., Li, B., \& Guo, Q. (2018). Naturalness preserved nonuniform illumination estimation for image enhancement based on retinex. IEEE Transactions on Multimedia, 20(2), 335-344.

[54] Li, M., Liu, J., Yang, W., Sun, X., \& Guo, Z. (2018). Structure-revealing low-light image 
48 H. Ackar et al./ Southeast Europe Journal of Soft Computing Vol.8 No.1 March 2019 (42-48)

enhancement via robust Retinex model. IEEE Transactions on Image Processing, 27(6), 28282841.

[55] Hossain, M. F., Alsharif, M. R., \& Yamashita, K. (2010, June). A New Image Enhancement Method Based on Nonsubsampled Contourlet Transform. In International Conference on Advanced Communication and Networking (pp. 74-80). Springer, Berlin, Heidelberg.

[56] Sivakumar, R., \& Mohan, E. (2018). High Resolution Satellite Image Enhancement Using Discrete Wavelet Transform. International Journal of Applied Engineering Research, 13(11), 98119815.

[57] Ma, J., Fan, X., Yang, S. X., Zhang, X., \& Zhu, X. (2018). Contrast limited adaptive histogram equalization-based fusion in YIQ and HSI color spaces for underwater image enhancement. International Journal of Pattern Recognition and Artificial Intelligence, 32(07), 1854018.

[58] Nnolim, U. A. (2018). An adaptive RGB colour enhancement formulation for logarithmic image processing-based algorithms. Optik, 154, 192-215.

[59] Samanta, S., Mukherjee, A., Ashour, A. S., Dey, N., Tavares, J. M. R., Abdessalem Karâa, W. B., ... \& Hassanien, A. E. (2018). Log transform based optimal image enhancement using firefly algorithm for autonomous mini unmanned aerial vehicle: An application of aerial photography. International Journal of Image and Graphics, 18(04), 1850019.

[60] Wan, M., Gu, G., Qian, W., Ren, K., Chen, Q., \& Maldague, X. (2018). Infrared image enhancement using adaptive histogram partition and brightness correction. Remote Sensing, 10(5), 682.

[61] Xiao, B., Tang, H., Jiang, Y., Li, W., \& Wang, G. (2018). Brightness and contrast controllable image enhancement based on histogram specification.
Neurocomputing, 275, 2798-2809.

[62] Loh, Y. P., Liang, X., \& Chan, C. S. (2019). Lowlight image enhancement using Gaussian Process for features retrieval. Signal Processing: Image Communication, 74, 175-190.

[63] Ahmad, R., \& Choubey, N. S. (2018). Review on image enhancement techniques using biologically inspired artificial bee colony algorithms and its variants. In Biologically Rationalized Computing Techniques for Image Processing Applications (pp. 249-271). Springer, Cham.

[64] Chen, J., Yu, W., Tian, J., Chen, L., \& Zhou, Z. (2018). Image contrast enhancement using an artificial bee colony algorithm. Swarm and Evolutionary Computation, 38, 287-294.

[65] Dhal, K. G., Ray, S., Das, A., \& Das, S. (2018). A Survey on Nature-Inspired Optimization Algorithms and Their Application in Image Enhancement Domain. Archives of Computational Methods in Engineering, 1-32.

[66] Cai, J., Gu, S., \& Zhang, L. (2018). Learning a deep single image contrast enhancer from multiexposure images. IEEE Transactions on Image Processing, 27(4), 2049-2062.

[67] Gomez-Ojeda, R., Zhang, Z., Gonzalez-Jimenez, J., \& Scaramuzza, D. (2018, May). Learning-based image enhancement for visual odometry in challenging HDR environments. In 2018 IEEE International Conference on Robotics and Automation (ICRA) (pp. 805-811). IEEE.

[68] Talebi, H., \& Milanfar, P. (2018, May). Learned perceptual image enhancement. In 2018 IEEE International Conference on Computational Photography (ICCP) (pp. 1-13). IEEE. 